\title{
Predicting mask distortion, clogging and pattern transfer for stencil lithography
}

\author{
Maryna Lishchynska ${ }^{\mathrm{a}, *}$, Victor Bourenkov ${ }^{\mathrm{a}}$, Marc A.F. van den Boogaart ${ }^{\mathrm{b}}$, \\ Lianne Doeswijk ${ }^{\mathrm{b}}$, Juergen Brugger ${ }^{\mathrm{b}}$, James C. Greer ${ }^{\mathrm{a}}$ \\ a Tyndall National Institute, Lee Maltings, Prospect Row, Cork, Ireland \\ ${ }^{\mathrm{b}}$ EPFL, Laboratoire des Microsystèmes, CH-1015 Lausanne, Switzerland
}

Received 11 May 2006; received in revised form 2 August 2006; accepted 4 August 2006

Available online 5 September 2006

\begin{abstract}
One of the ultimate tasks for stencil lithography is the ability to fabricate arrays of structures with controlled dimensions on the nanometer scale precisely positioned on a suitable surface. The race to shrink feature sizes requires the limits of conventional lithography to be extended to high-throughput, low cost, reliable and well-controlled processes of which stencilling is a promising candidate for nanoscale applications. Identifying, predicting and overcoming issues accompanying nanostencil lithography is critical to the successful and timely development of this technique for a wide range of potential applications. This paper addresses phenomena associated with stencil nanopatterning and presents the results of modelling and simulation studies for predicting the deleterious effects of mask distortion and clogging during pattern transfer. It is shown that degrading effects of stress-induced deformation of stencils can be dealt with via optimal design of corrugation structures which in turn reduce stencil deformation and significantly improves pattern definition. Modelling results are validated by comparison to experiment. The corrugation structures can be used to define practical design rules for fabrication of stable large area ("full scale") purpose-designed stencil membranes. The accurate modelling of the clogging phenomenon combined with gradually evolving stencil deformation, also presented in the paper, can be used for prediction of pattern distortion, to calculate maximum thickness of a deposited layer and/or for prediction of the stencil lifetime.
\end{abstract}

(c) 2006 Elsevier B.V. All rights reserved.

Keywords: Stencil lithography; MEMS; Modelling; Pattern transfer; Stress-induced deformation; Stencil stabilization

\section{Introduction}

Stencil lithography is one of the promising patterning methods for localized material deposition through ultraminiature shadow-masks (nanostencils) [1]. Shadow masking or stenciling has the advantage of allowing direct patterning of mesoscopic surface structures on a full-wafer scale by any material flux on a large variety of substrates. Being a direct or additive patterning technology it does not need cyclic processing steps seen, for example, in photolithography, a subtractive process. The stencil, which is effectively a thin

\footnotetext{
${ }^{*}$ Corresponding author.

E-mail address: marynal@tyndall.ie (M. Lishchynska).
}

membrane, allows for smaller aperture size and better uniformity of patterns over large surfaces which overcomes fabrication limitations usually associated with micro/nanopatterning. The method is also capable of achieving high pattern resolution with simultaneous high-throughput, giving access to nanometer-precise patterning with short processing times. The most common applications of stenciling have been patterning of printed circuit board features and interconnect technologies [2]. Potential applications of the stencil technique include (1) deposition on non-conventional and unstable substrate materials (i.e., bio-chemical, hydrophobic), (2) deposition of heterostructures (epitaxial, magnetic, complex oxides, piezoelectric materials), and (3) deposition of nanodevices onto CMOS [1-3]. 


\section{Stencil deposition principles, issues and previous work}

Stencil lithography is a direct or additive patterning technology, i.e., a controlled amount of a material is directly deposited through the stencil apertures onto the substrate (Fig. 1a). Deposition through stencils suffers, however, from deficiencies such as the clogging of apertures and the membrane deformation due to deposition-induced stresses. Consequently, the main challenges associated with application of the technique include reduced lifetime due to aperture clogging and excessive pattern distortion due to membrane deformation. Clogging occurs when the evaporated material accumulates on the stencil membrane and inside the stencil apertures (Fig. 1b). This phenomenon changes the shape of the aperture during the deposition process and leads to a distortion of the deposited pattern, eventually resulting in the complete closure of the aperture. Accumulation of material on the membrane and the subsequent deformation due to the deposition-induced stress (Fig. 1c) generally results in an increased gap between the substrate and the stencil resulting in pattern distortion and blurring.

Modelling and simulation techniques are of great assistance for assessing the stress-induced deformation of a stencil membrane, predicting pattern distortion and aiding stencil design optimization. Moreover, for correct process window definition, fabrication of efficient MEMS tools and consequently improved pattern definition, an accurate modelling of the membranes and a good understanding of related phenomena/effects is demanded. State-of-the-art modelling and simulation for nanolithography pattern transfer includes modelling the deformation of the stencil mask as well as prediction of pattern distortions. Reported in-plane deformation of masks, which are particularly susceptible to large distortions due to high membrane stresses, have been identified [4]. The results of finite elements method (FEM) simulations characterising distortions induced during the fabrication of electron-beam projection lithography masks report good agreement between experiment and simulation. Previous publications [5] suggest using voids as stress relief structures in order to reduce the stress-related in-plane distortion of stencil masks for ion-beam projection lithography. An extensive bibliographical review of the FEM methods applied to the analysis and simulation of nanolithography (electron-beam, optical, X-ray) and pattern transfer has been done by Mackerle et al. [6]. Other studies (e.g., Refs. [7,8]) concerned with the modelling of in-plane distortions of masks used in ion-beam lithography and X-ray lithography. Nevertheless, there appears to be no significant investigations into reducing out-of-plane deformations of the mask, which is vital for pattern definition by nanostencil lithography.

With regard to the clogging of stencil apertures during deposition, the characterization of the phenomenon has been performed in a number of papers [9-11]. Kölbel et al. $[10,12]$ introduced a method that reduces nanostencil aperture clogging by coating the stencil with self-assembled monolayers. While the effects of clogging were characterised by deposition of Er and Au layers though stencil apertures of dimensions as small as $20 \mathrm{~nm}$ in diameter [9]. However, no quantitative simulation studies on dependence of the shape of surface structures on deposition

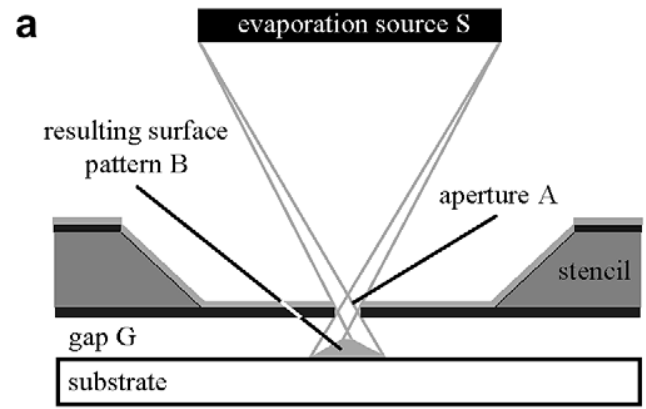

b
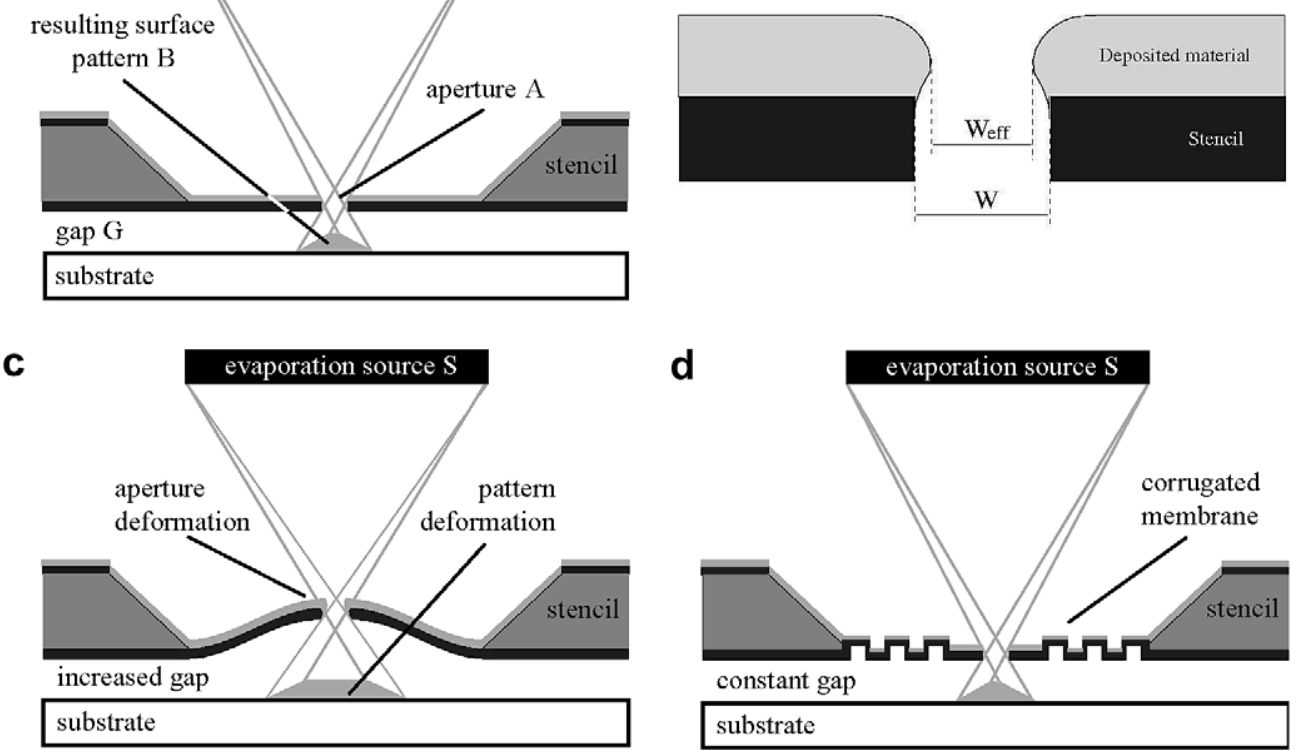

Fig. 1. Schematic of a membrane in close proximity to a substrate during evaporation: ideal stencil deposition (a); illustration of stencil mask aperture clogging, where $W$ is the initial aperture width, $W_{\text {eff }}$ is the effective width (b); realistic stencil deposition (deformed membrane); (c) and deposition through the corrugated stencil $(\mathrm{d})$. 
parameters (evaporation chamber geometry, materials), stencil geometry, stress-induced deformation and clogging effect (maximum thickness of deposited layer, stencil lifetime) have been published to date.

This paper aims to demonstrate the usefulness and accuracy of modelling techniques for predicting the extent of membrane deformation, for geometry optimization by means of incorporating stabilization structures into the stencil (Fig. 1d), and for predicting pattern distortion due to the combined effects of clogging and stencil deformation.

\section{Modelling effects of deposition-induced stress in MEMS stencils}

\subsection{Origins and consequences of deposition-induced stress}

Film deposition processes employed in current manufacture of microelectromechanical systems (MEMS) generate stress within both the substrate and the deposited film.

Excessive film stresses can lead to substantial deformation of structures, thus rendering a device unusable. Clear understanding of the underlying mechanics and accurate assessment of the deformation caused by film stress is critical for developing controllable processes and producing high quality devices.

Excessive residual stresses, usually undesirable in MEMS structures, arise from a variety of sources. When a film on a substrate is subjected to a temperature change during the evaporation, a difference in the thermal expansion coefficients of the two materials results in thermal stresses $\sigma_{\text {th }}$ [13] (Fig. 2). All other stresses originating "from within" thin films during the deposition process fall in the category of intrinsic stresses $\sigma_{\text {int }}$. Various physical factors responsible for the intrinsic stress have previously been studied and explained in the literature $[14,15]$. Most of these factors are summarised in [13] as follows:

- differences in the expansion coefficients of film and substrate;

- lattice mismatch between substrate and film (Fig. 3);

- incorporation of atoms (e.g., residual gases) or chemical reactions;

- variation of the interatomic spacing with the crystal size;

- recrystallization processes;

- microscopic voids and special arrangements of dislocations;

- phase transformations,

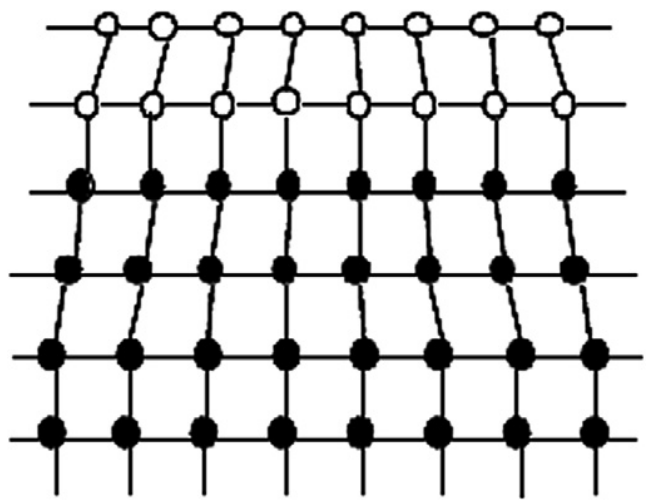

Fig. 3. Schematic of strain in thin layer of material due to lattice mismatch.

where the first two causes are believed to be dominant. In soft, low melting point materials (e.g., aluminium), bulk diffusion tends to relax the internal stresses and to prevent their accumulation [16]. In hard, higher melting point materials (e.g., chromium) deposited at low temperatures, intrinsic stresses accumulate and tend to dominate over thermal stresses.

The magnitude of thin film stress depends on film thickness and on the fabrication process used [17]. Therefore, it is not reliable to extrapolate stress values obtained under some conditions to that at others. A truly quantitative theory of thin film stress is yet to be developed, thus measurement remains the only available means to determine residual stress. Thin film stress is commonly quantified using a wafer-bending technique involving measurement of the curvature of a substrate of known dimensions before and after film deposition [18]. Importantly, these measured values of residual stress are the stress levels in the film (not in the structures). Beams and cantilevers from the films often have a lower stress due to relaxation in the width direction [19].

Following release of a device, residual stresses in the elastic structural layers are partially relieved by deformation [20]. The extent of deformation is strongly dependent on process details and on the geometry of the structure. Stress gradients through the thickness of a micromachined film are particularly undesirable as they cause a significant deformation of a structure. This is especially relevant in the case of bilayered MEMS where different residual stresses occur in different layers. As material elastic properties and residual stresses differ in layers, each layer tends to contract (tensile stress) or expand (compressive stress) by

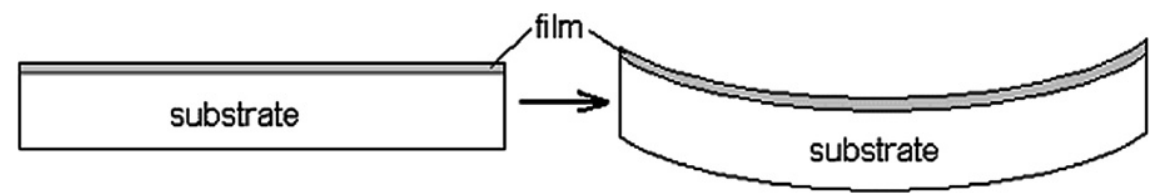

Fig. 2. Origins of thin film stresses: film under residual tensile stress due to CTE mismatch. 
a different magnitude thus resulting in deformation/bending of the structure (Fig. 4).

The performance of many MEMS devices and particularly nanostencils is significantly restricted by the adverse effects of residual stress. The following sections address this problem and aim to find optimal methods for reducing the detrimental effects (deformation) thus stabilizing the stencils.

\subsection{Modelling stress-induced deformation in bi-layered unstabilized (planar) cantilevers}

Modelling of full size complex apertured membranes (stencils) is a challenging task. Therefore, in order to simplify the first approach and to validate the Coventor FEM modelling tool [21], cantilever-like membranes were firstly considered. The cantilever is a basic design component for MEMS found within numerous applications. Each cantilever was modelled as a bi-layered structure (Fig. 4) comprised of a 500-nm thick SiN membrane layer and a 50-nm thick $\mathrm{Cr}$ deposition layer. Experimentally measured values of biaxial tensile intrinsic stress of $1230 \mathrm{MPa}$ incorporated in Cr layer and $200 \mathrm{MPa}$ in SiN layer of the cantilever were introduced into the simulations.

Thin film properties of materials used in MEMS technology are strongly dependent on processing conditions. Therefore, there is an evident uncertainty in published elastic material properties data of both SiN and Cr. Material properties of $\mathrm{SiN}$ are particularly affected as there exists a high discrepancy in published Young's modulus values $(E \approx 130-385 \mathrm{GPa})$ [22]. Furthermore, addition of metallic or polymeric coatings on the cantilever surface can significantly modify the cantilever's elastic properties [23]. As no characterization for the thin film properties of the materials used has been performed in this work, material properties from processes similar to the ones employed here were used in this study (Table 1).

Significant curling of the simulated plane (unstabilized) cantilevers is observed. The results are validated via comparison with measured deflections (Fig. 5a). A good agreement between simulated and measured data is evident validating the Coventor software tool. Fig. 5b shows the deformation observed in micromachined cantilevers.

\subsection{Reduced deformation of corrugated cantilever membranes}

In order to overcome or at least to minimise the effects of membrane deformation, it was proposed [26] to mechan-
Table 1

Material properties used in simulation study

\begin{tabular}{llll}
\hline Material & Young's modulus, GPa & Poisson's ratio & Reference \\
\hline $\mathrm{SiN}$ & 276 & 0.27 & {$[24]$} \\
$\mathrm{Cr}$ & 277 & 0.3 & {$[25]$} \\
\hline
\end{tabular}

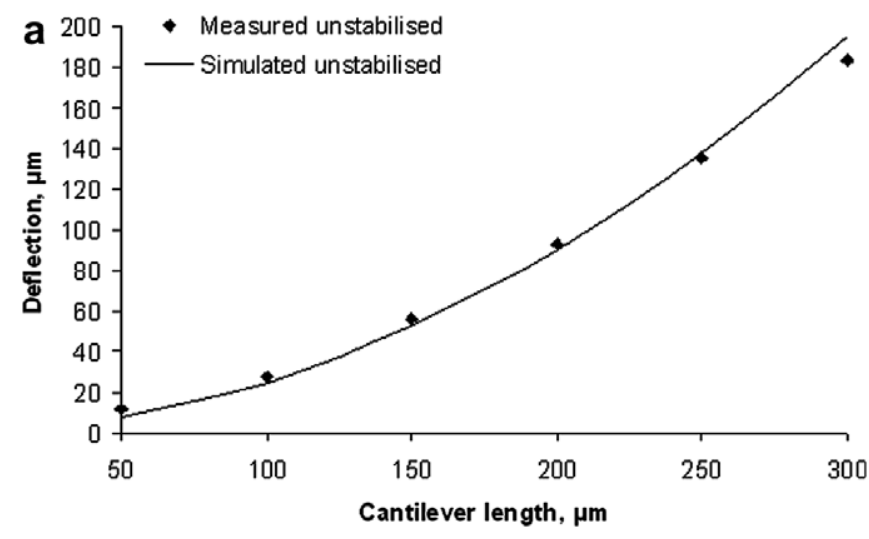

b

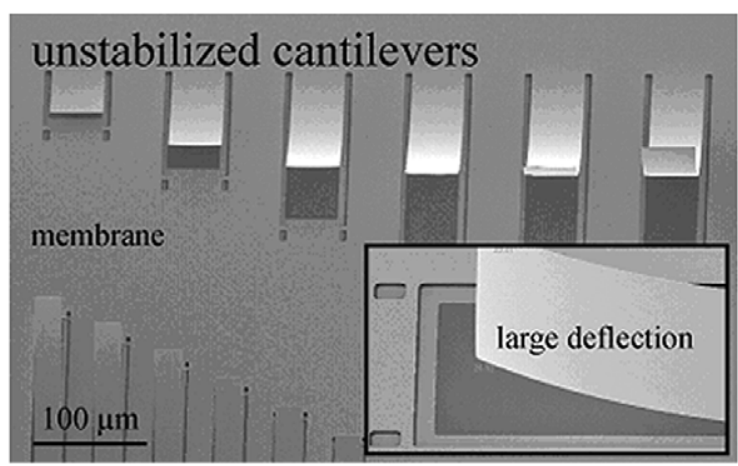

Fig. 5. Deflection values for unstabilized (plane) cantilevers: simulated (solid line) versus measured (dots) (a) and SEM image of a membrane containing unstabilized cantilevers after deposition of 50-nm thick $\mathrm{Cr}(\mathrm{b})$.

ically reinforce and thus stabilize the membranes. The improved micro/nanostencils incorporate in situ, local stabilization structures increasing their moments of inertia, which are the structural property directly related to stiffness or deformability (Figs. 1c and 6). A basic flow of the fabrication process is given in [26] where stabilizing effect of corrugation on membrane deformation is computationally and experimentally confirmed. The present work is concerned with predicting the optimal geometry for corrugation structures and predicting stencil deformation, clogging and subsequently definition of deposited pattern. Various geometries of stabilization structures studied in this work are shown in Fig. 7.

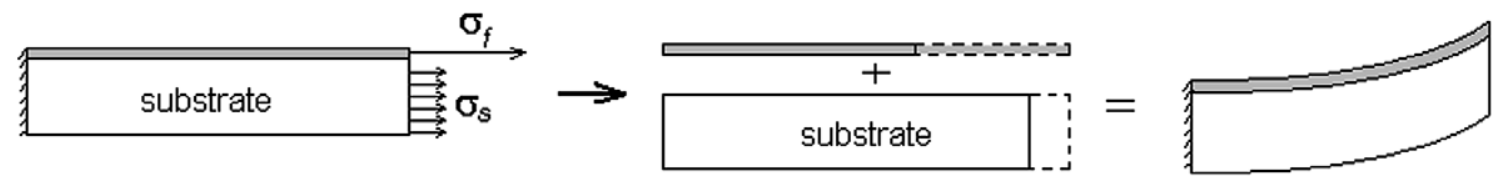

Fig. 4. Schematic of a bi-layered cantilever deformation due to high residual stress $\sigma_{\mathrm{f}}$ in additional film layer. 

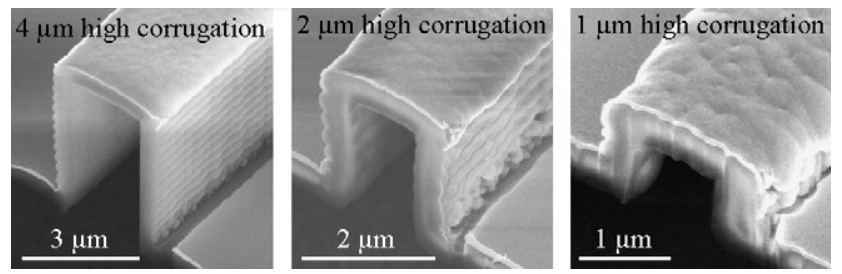

Fig. 6. SEM images of cross-sections of micromachined cantilevers with corrugation of various heights.

To analyze the mechanical stability of reinforced cantilevers, all structures depicted in Fig. 7 were modelled and simulated with finite element models (FEM) as implemented within Coventor [21]. A series of cantilever-type corrugated membranes with 1-4 stabilization rims with heights varying from $1 \mu \mathrm{m}$ to $4 \mu \mathrm{m}$ were fabricated. These stabilized membranes were coated with a 50-nm thick $\mathrm{Cr}$ thin film using electron-beam evaporation (Alcatel, EVA 600). A supplementary reference wafer was used for the measurements of the thin film residual stress by means of wafer curvature (Tencor). The deflection of cantilever-type membranes was determined by measuring the maximum deflection of each cantilever by both Scanning Electron Microscopy (SEM, LEO 1550) and optical surface profiling (Veeco, NT1100). A comparison of measured and simulated vertical deflection values for different cantilever lengths, deposition thicknesses and stabilization types is shown in Fig. 8 (additional data is presented in [26,27]) and indicates a good correlation, again confirming the FEM modelling tool as a reliable means of prediction of stress-induced deformation.

A comparative simulation study on optimization of the geometries required for stabilization of cantilevers was undertaken in this work. It was confirmed that stabilization structures significantly reduce (up to 96\%) out-of-plane deformation of the cantilever membrane. The results also show that the box-type stabilization is the most optimal among the geometries considered (Fig. 9). Although the quantitative effects of rim- and box-type corrugation on reduction of deformation are close, introducing only parallel rims changes the deformation state at cantilever tip

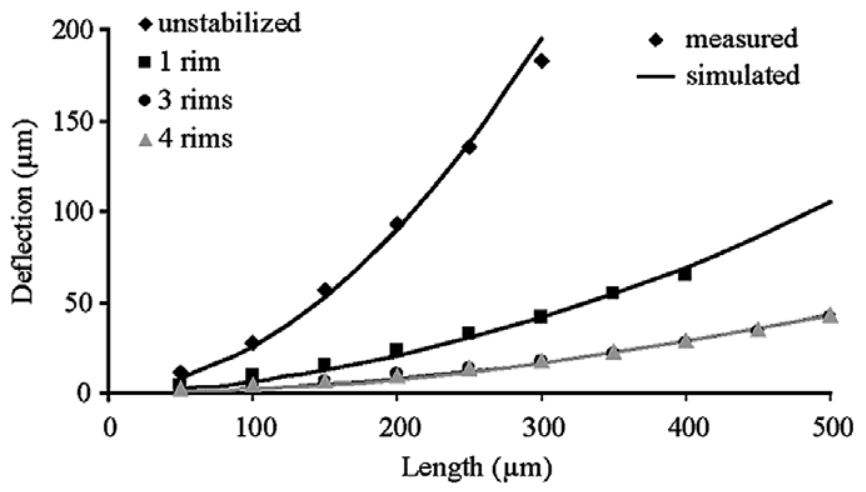

Fig. 8. Plot of simulation results (solid lines) versus measurement data (dots) for corrugated cantilevers with a variable number of $1-\mu \mathrm{m}$ high rims.

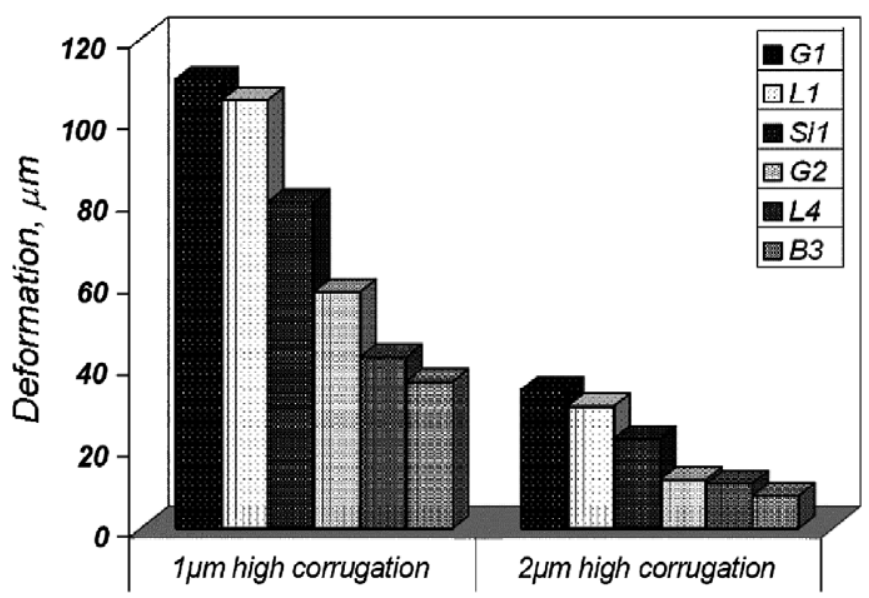

Fig. 9. Comparison of the effect of different geometries of corrugation on cantilever deformation (simulation results). Corrugation rims of two heights $(1 \mu \mathrm{m}$ and $2 \mu \mathrm{m})$ were studied.

causing lateral bending (cantilever corners curl up significantly, see Fig. 10). This effect can be prevented or reduced by introducing an extra rim at the cantilever tip (Fig. 7, corrugation B3). Thus, deformation profile evolves correspondingly to corrugation geometry. This implies that in
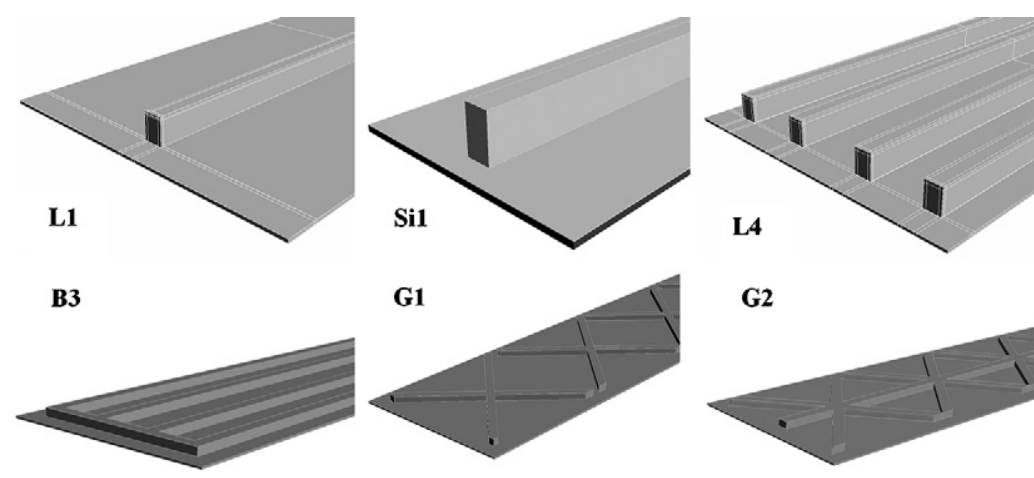

Fig. 7. Proposed stabilization geometries: 1 hollow rim (L1), bulk Si (Si1), 4 rims (L4), hollow box (B3), grid-1 (G1) and grid-2 (G2). Number of rims varies from 1 to 4 with height equal 1,2 or $4 \mu \mathrm{m}$. 


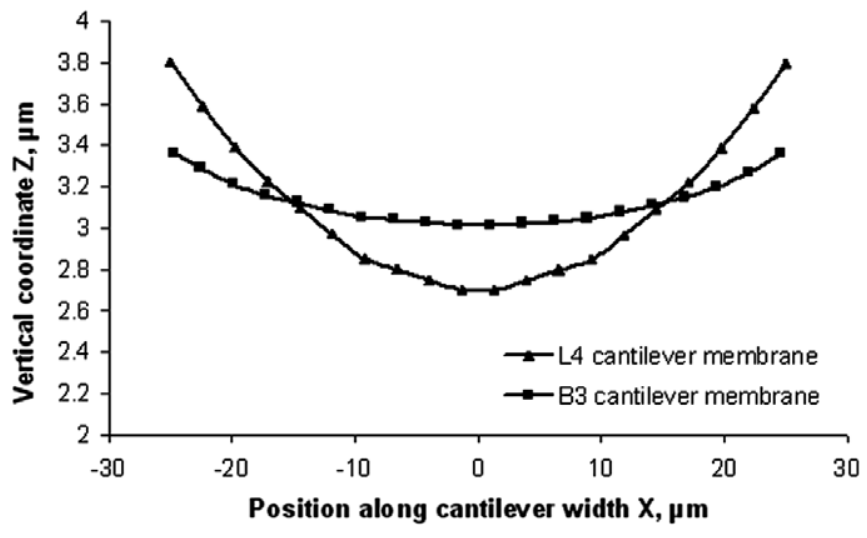

Fig. 10. Cross-section profiles of deformed L4 and B3 cantilevers.

practice, design of stabilization structures for real stencils is an iterative process whereby a series of "modelling the geometry of stabilization structures - simulating the defor- mation" cycles may have to be undertaken before a target reduction of deformation is reached.

A conclusion drawn from the above-results is that the largest reduction in deformation, and improvement in pattern definition, are achieved with corrugation rims positioned perpendicular to the direction of increasing deformation or "deformation isocontours" (Fig. 11a). This guideline can be used as a practical design rule for stabilization of complex apertured membranes (Fig. 11b), thus for optimization of the design before implementation and improving the dimensional control over the deposited patterns/structures.

It is worth mentioning that all above-described methods for stabilizing the membranes cannot achieve zero distortion as the stabilization structures do not extend to the edges of the cantilevers. This limitation is imposed by the design rules currently needed for mask fabrication due to the requirement of a separation $(2-5 \mu \mathrm{m}$ depending on a
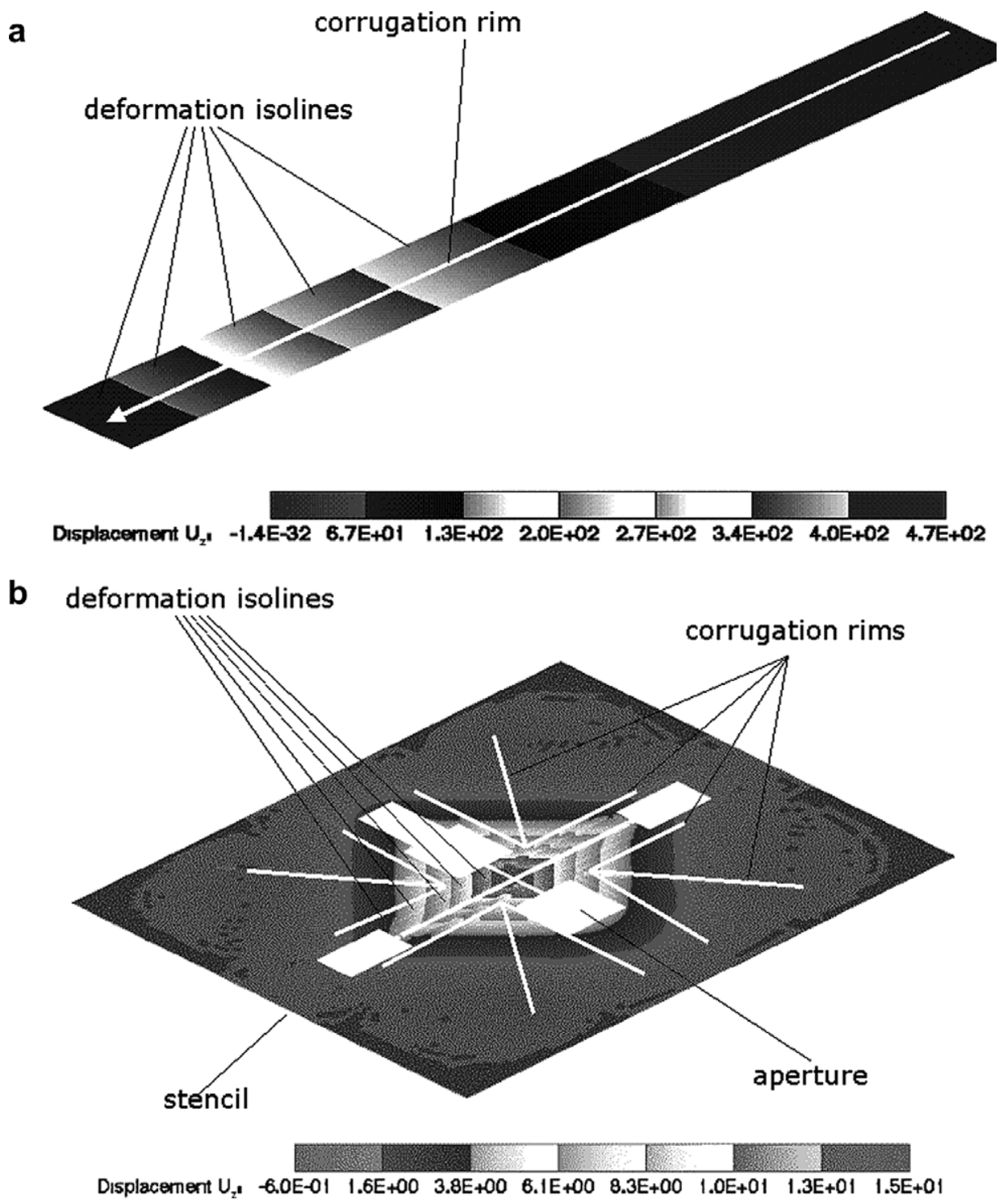

Fig. 11. Deformation isocontours in a cantilever membrane (a) and in apertured membrane/stencil (b). Bold white lines suggest the direction where most effective corrugation structures should be located. 
case) from the membrane edge in order to assure a sufficient evaporation angle, leaving edges of the membrane to curl to some extent. Nevertheless, a substantial reduction in deformation of the membranes enables multiple usage of designed stencils and provides better control over the pattern definition, which is shown in the following section.

\subsection{Predicting stencilled pattern distortion due to membrane deformation: before and after introduction of corrugation}

Stencil deformation leads to an increased gap between the stencil and the substrate which causes significant pattern distortion termed blurring (broadening, edge sloping) (Fig. 1c). Blurring occurs due to the geometry of the deposition system set up (surface is exposed to the direct material beam) and surface diffusion. Naturally, both these factors have more tangible effect on pattern transfer with smaller deposited structures. From purely geometrical considerations as depicted in Fig. 12, vertical stencil deformation, $\delta$, translates into broadening of the pattern, $b$, according to the following formula:

$b \approx \frac{G_{\text {effective }} S}{D}=\frac{(G+\delta) S}{D}$,

$G$ is the gap between the stencil and the substrate, $S$ is source size, $D$ is the distance from the source to the stencil, $b=B-W, W$ is aperture width corresponding to the ideal pattern width.

As an example, consider a membrane with apertures designed to allow for deposition of $5 \mu \mathrm{m}$ wide lines (Fig. 13). One can estimate the distortion of the deposited pattern (blurring in this case) induced by stencil deformation and the improvement in resolution achieved via membrane corrugation. Fig. 14a presents values of pattern broadening estimated via formula (1) for unstabilized and stabilized cantilever membranes. It can be seen that corrugation reduces pattern broadening by nearly two orders of magnitude, reducing it to submicron values. Such a low degree of blurring forms only a fraction of the intended ideal size (width) of the pattern and sets the tolerance for pattern

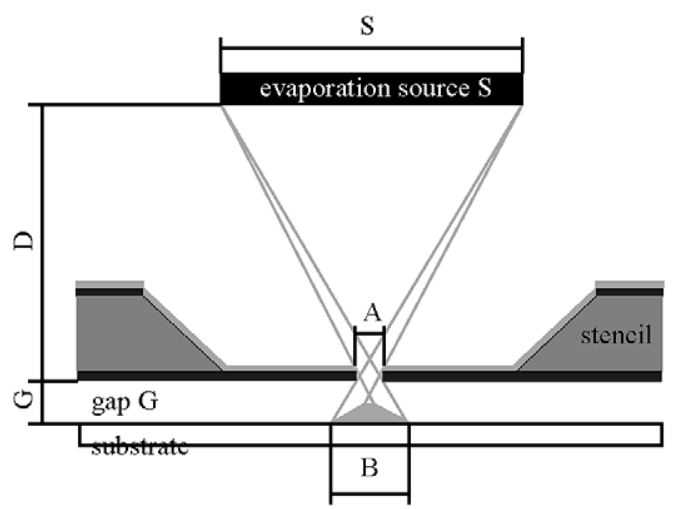

Fig. 12. Schematic of pattern broadening due to geometrical factors (finite distance to the source $D$, non-zero gap $G$, source size $S$, aperture width $A$, deposited pattern width $B$ ). a
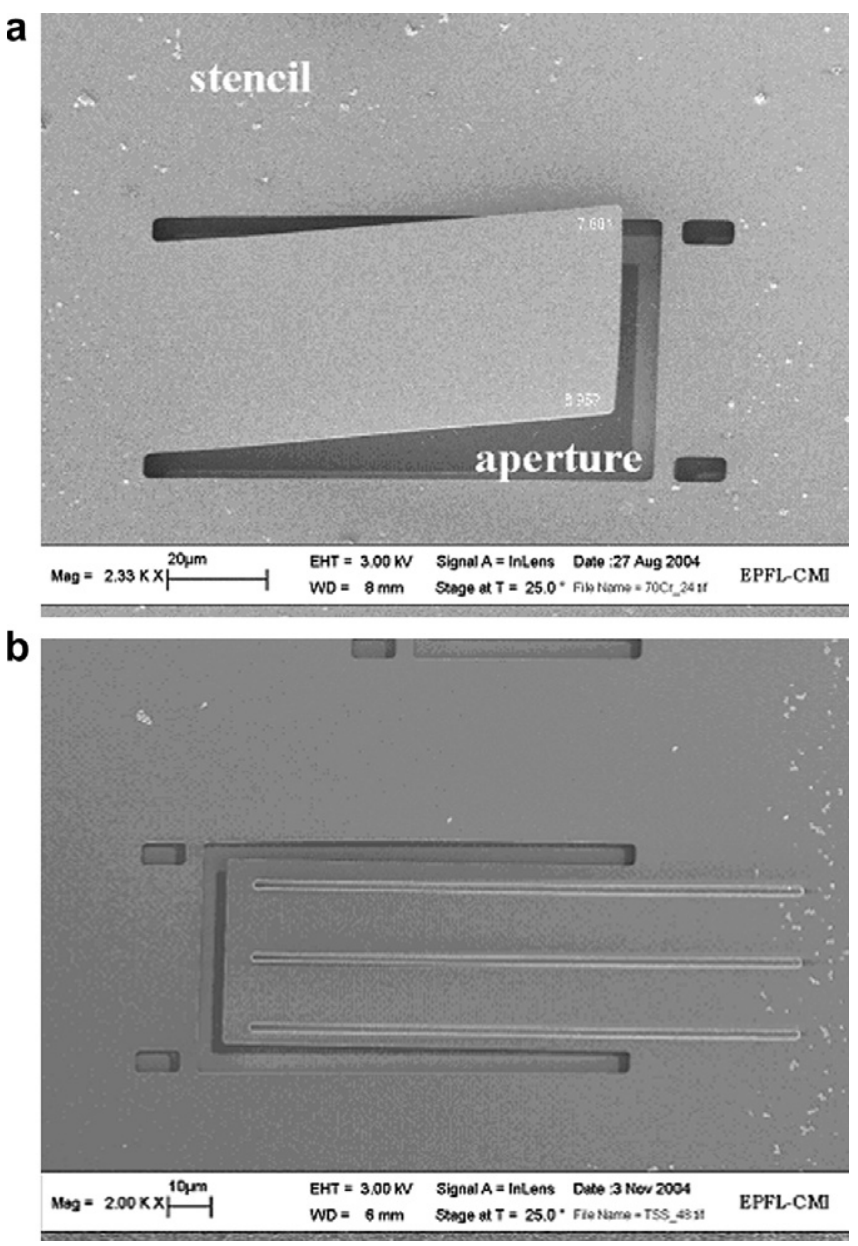

Fig. 13. SEM image of the cantilever-like membranes in close proximity to the substrate: unstabilized stencil, increased gap $G$ is evident; stabilized (corrugated) stencil, no significant change in the gap.

transfer. The effect of corrugation on deposited structure profiles was also modelled using a physical vapour deposition (PVD) tool - DepoSim - developed in this work [28] (described in the next section). A significant improvement in edge definition (reduced blurring) is achieved (Fig. 14b).

In order to assess experimentally the effect of membrane corrugation on deposited pattern definition, microfabricated cantilever-like stencils were removed from a substrate and the surface structures were analyzed by scanning electron microscopy (SEM). Dynamic Force Microscopy (DFM) surface profile scans were taken to characterise the deposition profile and edge acuity. Fig. 15 shows images of $45-\mathrm{nm}$ thick $\mathrm{Cr}$ surface structures deposited through both an unstabilized stencil membrane (Fig. 15a) and a corrugated stencil membrane (Fig. 15b). The SEM image and the DFM images in Fig. 15a show reduced pattern definition and reduced contrast between the deposited $\mathrm{Cr}$ and the Si substrate on the larger stencil aperture. The line scan in Fig. 15a shows the pattern edge definition in detail. The difference between the pattern edge definition for uncorrugated and corrugated stencils is clearly visible, indicated by the different edge slope (blurring). The SEM image and the DFM images of the surface structures 

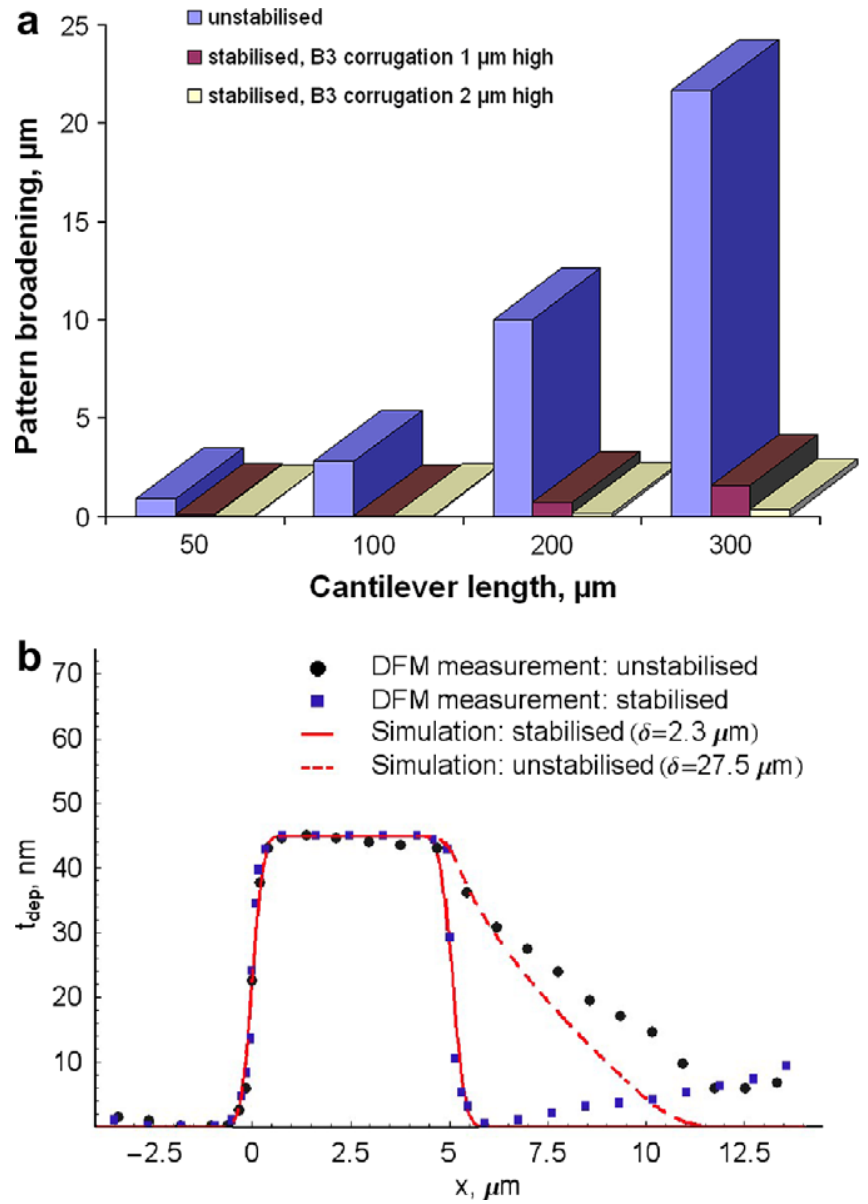

Fig. 14. Plot of pattern broadening values for unstabilized and stabilized cantilever membranes of various lengths (a) $(D=0.45 \mathrm{~m}, S=0.05 \mathrm{~m}$, assumed gap is zero) and comparison of experimental and simulated profiles of deposited structures for unstabilized and stabilized membranes (b).

deposited through the stabilized stencil (Fig. 15b) show excellent pattern definition and contrast between the deposited $\mathrm{Cr}$ and $\mathrm{Si}$ substrate. The line scan in Fig. 15b shows the symmetrical pattern edge definition on both sides indicating a stable membrane. These results confirm that the corrugated membranes significantly reduce the out-of-plane deformation resulting in an improved stencilled pattern due to a reduction in the increase in gap distortion. Good correlation between measured and simulated pattern profiles validates numerical models as suitable for prediction of stenciled pattern distortion.

\section{Modelling clogging phenomenon and predicting stencil lifetime}

\subsection{Clogging phenomenon: empirical and theoretical characterization}

The influence and effect of clogging on the shape of deposited structures and the re-usability of the masks are important issues of nanoscale stencil patterning. Nanoscale apertures are easily clogged as the evaporated material is a
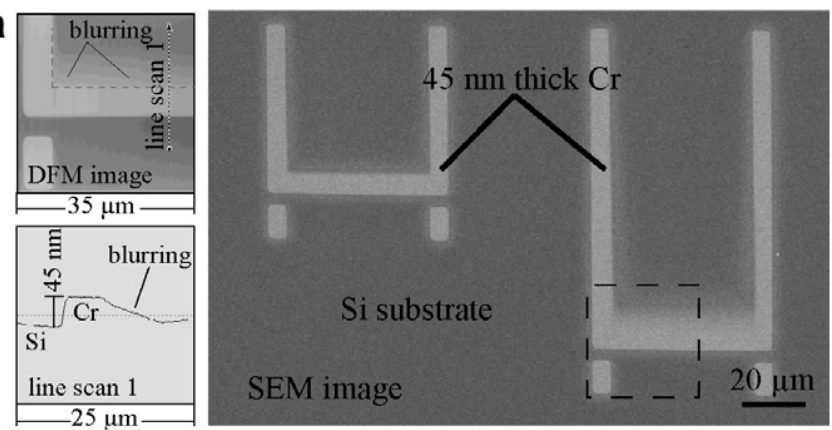

b

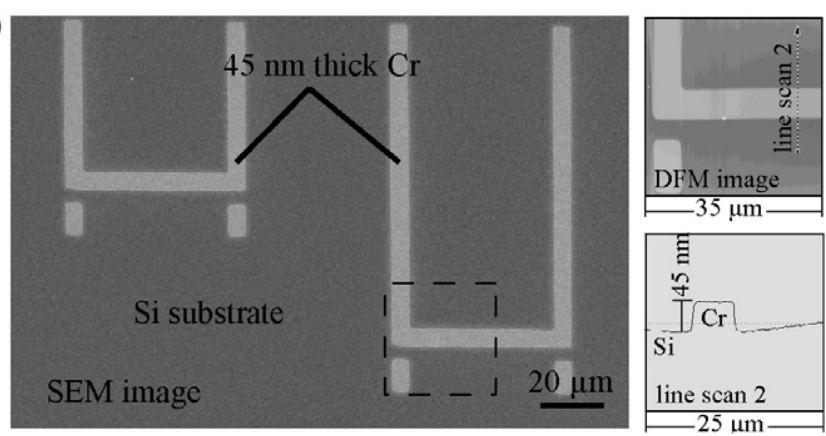

Fig. 15. SEM images and dynamic force microscope images (DFM) of surface structures made by the stencil deposition process: for unstabilized stencil membranes (a), both SEM and DFM images show pattern blurring of the deposited Cr surface pattern and for stabilized membrane (b) both SEM and DFM images show excellent pattern definition for stabilized stencils.

also deposited on the sidewalls of the stencil apertures. This phenomenon reduces the aperture openings during the deposition process, eventually closing the stencil apertures completely. It also results in sloped edges of the deposited structures and thus a non-uniform thickness of deposited layer (Fig. 16). Stencil masks can be cleaned by wet-chemical etching or dry plasma cleaning of the unwanted deposited material [29]. Clogging during the deposition imposes a limit to the amount of material that can be deposited in a single process step using stencils.

Experiments have been performed to investigate the degree of clogging of membrane apertures during thin film evaporation. Fig. 16c provides an example of metal deposition and the resultant clogging in microstencils. The EPFL stencil in Fig. 17 had originally $2.5 \mu \mathrm{m}$ wide apertures. This width has been reduced to $2.4 \mu \mathrm{m}$ after deposition of $110 \mathrm{~nm}$ thick $\mathrm{Cu}$ layer and to a $1.8 \mu \mathrm{m}$ width after $775 \mathrm{~nm} \mathrm{Cu}$ deposition.

Simulations of physical vapour deposition (PVD) through stencil mask apertures were performed using the Silvaco process simulation tools Athena/ELITE and DevEdit [30] and the PVD simulation code DepoSim [28]. The ELITE deposition models use the string algorithm limiting the simulations to two dimensions. The process simulator Athena has the ability to model the arrival of vapour flux from different directions. To take into account geometrical parameters of the experimental deposition setup, a simple computer program DepoSim has been written which calculates material deposition angles and the dependence of 
a

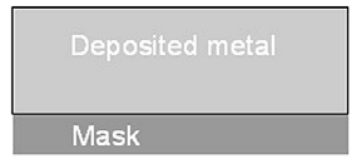

Mask

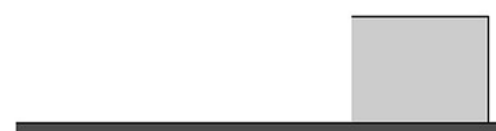

Substrate
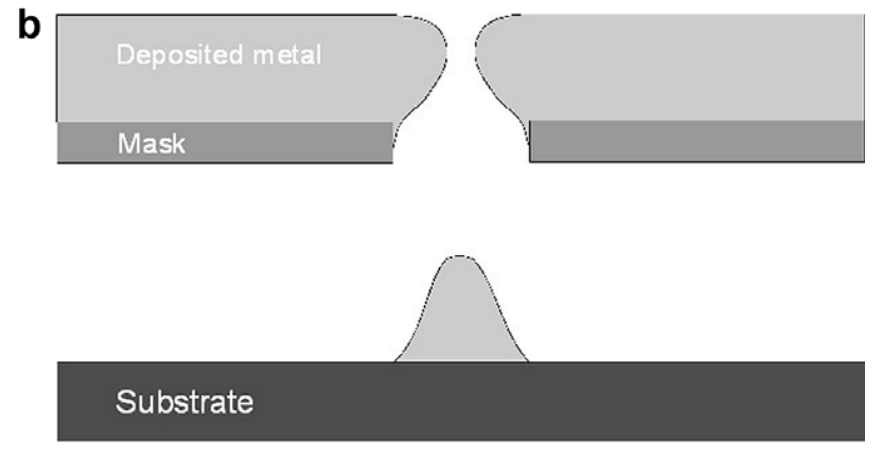

C

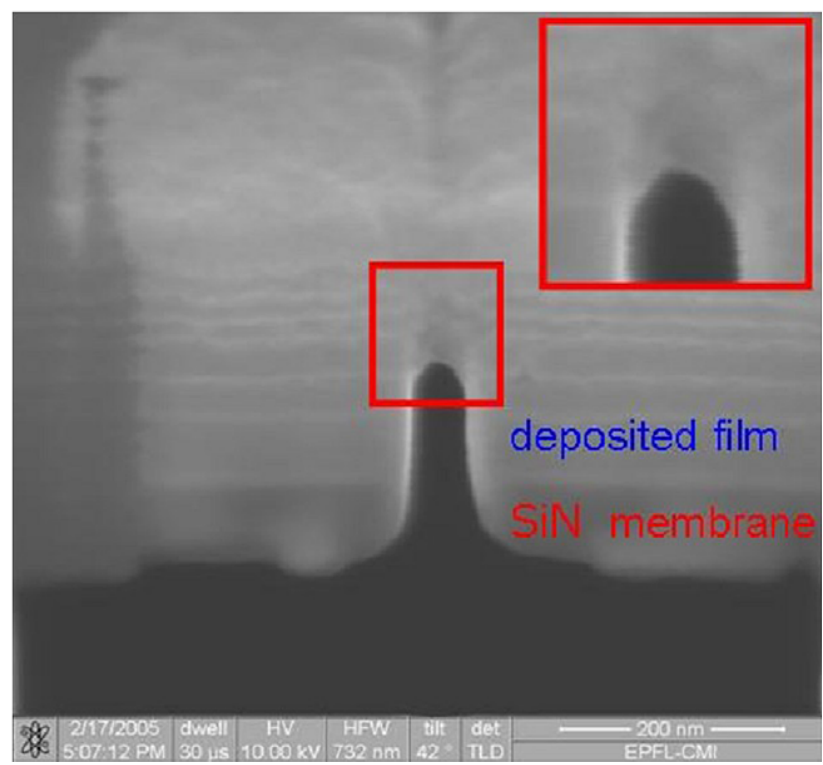

Fig. 16. Illustration of metal deposition though a mask: ideal case, no aperture clogging (a), aperture clogging (b), and the SEM image of a clogged nanoslit (c). deposition rates on the deposition angle. Process simulations are used to characterise the shape of the deposited structures and the clogging phenomena.

The simulations show that clogging rate depends on the angle distribution of the incident material flux, the magnitude of the surface diffusion, and the material sticking probability. The reduction of the aperture width (clogging) may be partly caused by the material deposition on the sidewalls of the aperture. Thus, if an incidental angle of the material arriving at the stencil mask plane is different from normal, some material will be deposited at the sidewalls. In the case investigated here (low-pressure electron beam evaporation PVD), the mean free path of the deposited particles exceeds the size of the deposition chamber, so the particles arrive at angles very close to the normal angle if the mask is positioned directly under the source. Our simulations show that the narrow angle distribution of the flux in this case means that the amount of the material directly deposited on the aperture sidewalls is very small. The reduction of aperture width due to direct material deposition on the sidewalls can be estimated using following geometric formula:

$\Delta W_{a}=\frac{t_{\mathrm{dep}} S}{2 D}$

where $t_{\text {dep }}$ is the thickness of the material deposited on the plane which is perpendicular to the line between the source and the stencil mask. For the experimental system setup considered here, the ratio $\mathrm{S} / \mathrm{D}=0.1$, so the reduction of aperture width due to deposition of $1000 \mathrm{~nm}$ of material is only $50 \mathrm{~nm}$ (Fig. 18).

In low-energy regime, typical for physical vapour deposition, all metal atoms are adsorbed independently of their impact angle. Studies of sticking probability dependence on the impact angle and the kinetic energy of impinging atoms are reported in $[31,32]$. The magnitude of the evaporationinduced kinetic energy, $E_{\mathrm{kin}}$, is generally related to the material vapourization temperature, $T_{v}$, by the Boltzmann temperature equation $[33,34]$ :

$E_{\text {kin }}=\frac{2}{3} k T_{v}=\frac{1}{2} m v^{2}$,

where $m$ is the particle mass, and $v$ is the particle velocity.

Actual particle (atom) velocities may be somewhat greater than calculated using Eq. (2). Measurements of
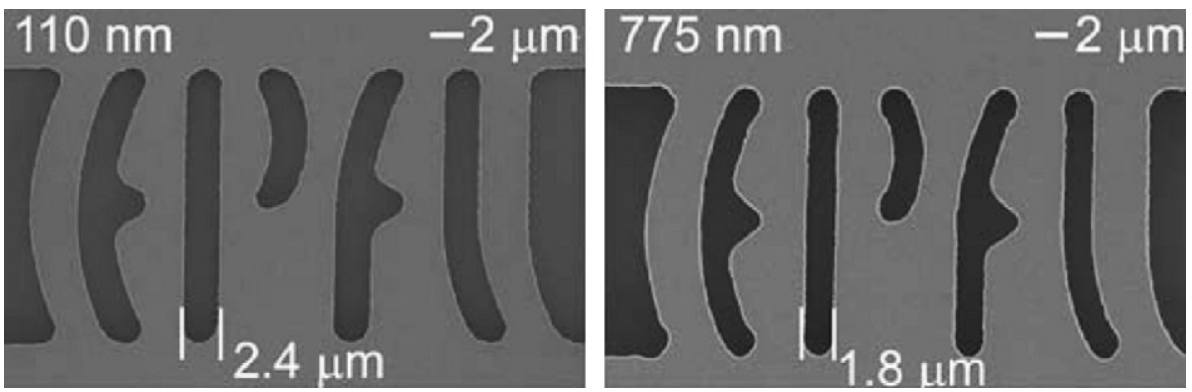

Fig. 17. SEM images of EPFL stencil after deposition of $110 \mathrm{~nm} \mathrm{Cu}$ (a) and $775 \mathrm{~nm} \mathrm{Cu}$ (b). 


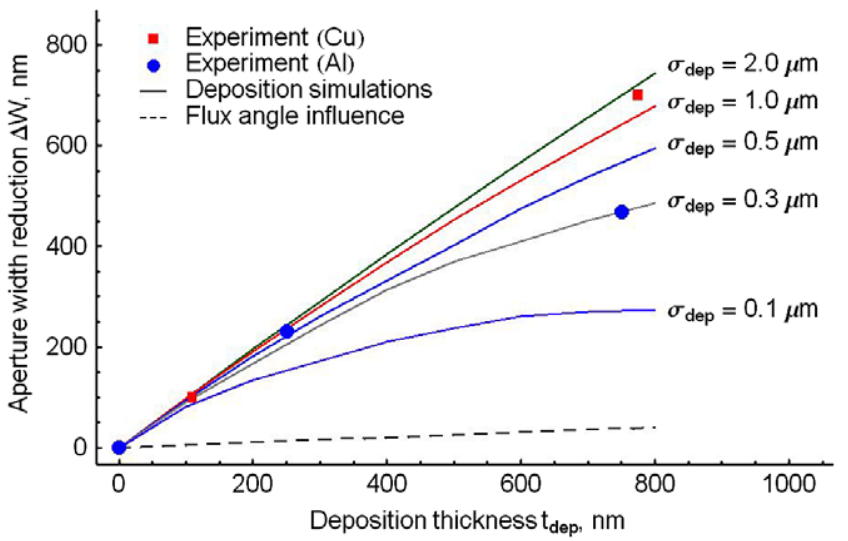

Fig. 18. Reduction of stencil aperture width versus deposited material thickness.

uranium, titanium and copper mean atomic velocities by Asano et al. [35] shows that the mean velocity of each atom type exceeds the predicted values. The velocities were 2.1 , 1.6, and 1.3 times greater than predicted by Eq. (2) for uranium, titanium, and copper, respectively. However, even taking into the account these greater velocities, the kinetic energy of atoms deposited by PVD is of the order of few tenths of eV. Molecular dynamic studies (e.g. [31]) show that kinetic energies of impinging atoms are not sufficient for them to leave the strongly attractive adsorption energy potential well. For example, the adsorption well depth given by Stumpf et al. [36] for aluminum surfaces is 3.10 $\mathrm{eV}$ on $\mathrm{Al}(111), 3.77 \mathrm{eV}$ on $\mathrm{Al}(100)$, and $3.89 \mathrm{eV}$ on $\mathrm{Al}(110)$. Since the kinetic energy of the atoms is much smaller than the adsorption energy, the probability of the atom leaving the surface is small. Thus, a unity sticking probability can be assumed in simulations of metal PVD.

The Silvaco process simulator uses an analytical approach to calculate surface diffusion through a normalized Gaussian distribution:

$N_{\text {dep }}=\exp \left(-\frac{x^{2}}{\sigma_{\text {dep }}^{2}}\right)$

where $x$ is the point at which material is deposited, $\sigma_{\text {dep }}$ is the diffusion parameter. Function $N_{\text {dep }}$ simulates the position dependence of the material deposition rate. Surface diffusion is an important factor in the occurrence of clogging. Due to the surface migration, the deposited material is re-distributed on the aperture sidewalls thus facilitating clogging. Simulations were performed to assess the influence of the surface diffusion parameter $\sigma_{\text {dep }}$ on the effective width of the stencil aperture. The simulated deposition of a material of thicknesses in the range from 0 to $1000 \mathrm{~nm}$ was carried out on a microstencil with an aperture width of $2.5 \mu \mathrm{m}$ (Fig. 18). The surface diffusion parameter $\sigma_{\text {dep }}$ was varied from $0.1 \mu \mathrm{m}$ to $2.0 \mu \mathrm{m}$. The simulation results shown in Fig. 18 indicate that an increase in the diffusion parameter leads to faster aperture width reduction (clogging).
Experimentally observed aperture width reduction is also shown in Fig. 18 for $\mathrm{Cu}$ and $\mathrm{Al}$ depositions. The comparison between simulated and experimental data suggests that in order to account for the experimentally observed clogging the surface diffusion parameter $\sigma_{\text {dep }}$ should be set at approximately $0.3 \mu \mathrm{m}$ and $1.5 \mu \mathrm{m}$ for the simulation of $\mathrm{Al}$ and $\mathrm{Cu}$ deposition, respectively.

Results on the clogging phenomenon can be used to estimate the amount of material, max $t_{\text {dep }}$ that can be deposited through a stencil before the mask becomes unusable. Such value of max $t_{\text {dep }}$ defines the stencil lifetime. Thus,

$\max t_{\mathrm{dep}}=f\left\{\frac{\Delta W_{\max }}{W_{0}}, \sigma_{\mathrm{dep}}, z_{i}\right\}$

where $\Delta W_{\max }$ is the maximum allowed pattern narrowing, $W_{0}$ is the initial intended pattern width (aperture width), $z_{i}$ represents other influential parameters related to stencil application, pattern density, stencil aspect ratio (defined as feature height to aperture width). For instance, analyzing Fig. 18 one can conclude that if pattern/aperture narrowing of up to $20 \%$ is acceptable, only $500 \mathrm{~nm}$ of $\mathrm{Cu}$ can be deposited through the mask.

\subsection{Combining effects of mask deformation and clogging on pattern definition}

The effects of the stencil deformation on the shape of deposited structures are difficult to simulate using commercially available process simulators such as the one used in this work (Silvaco Athena), because the evolution of the mask deformation during the deposition cannot be incorporated into simulations. For this purpose, a customised two-dimensional PVD simulation code DepoSim [28] was developed in this work. Since the mean free path of evaporated particles (in the case of low-pressure electron beam evaporation PVD) exceeds geometrical dimensions of the deposition chamber, the particles can be considered travelling along straight lines from the source to a particular point of the substrate. This physical phenomenon is simulated using the ray-tracing algorithm incorporated in DepoSim. The surface diffusion is calculated through a normalized Gaussian distribution (Eq. 4). The DepoSim tool simulates material transport through confined geometries in a PVD process taking into account the gradually increasing deformation of the stencil. Fig. 19 illustrates the profiles of a deposited structure and of the stencil mask; $t_{\text {dep } i}$ is the thickness of the $i$ th deposited layer, $S_{i}$ is the stencil profile after deposition of $t_{\mathrm{dep} i}$ of $\mathrm{Cr}$ (accounting for the aperture clogging).

Using simulation results on the mask deformation and the material deposition parameters, it was possible to predict the shape of structures deposited through a mask which deforms during the deposition. The code was also used to model the effect of corrugation on deposited structure profile (Fig. 14b). The simulation of deposited pattern blurring due to the mask deformation shows good correlation with the experimental data; compare to Fig. 14b. 

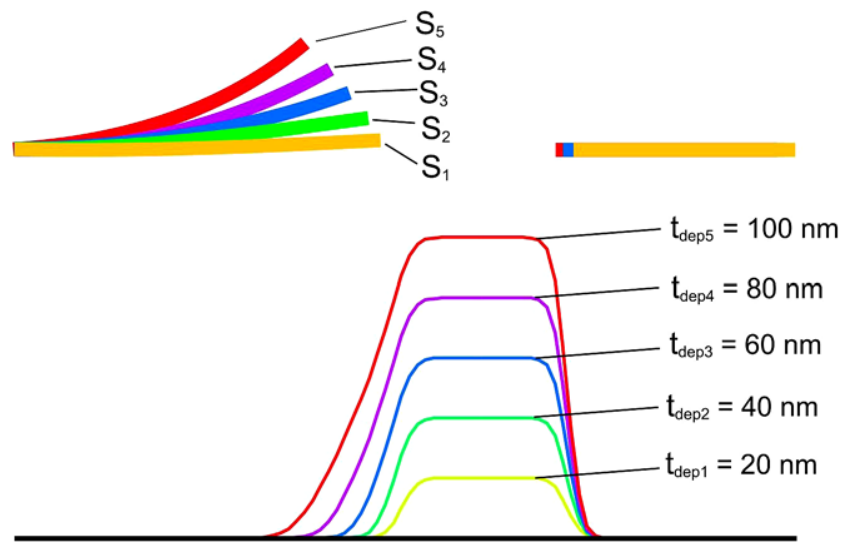

Fig. 19. Schematic of simulated stencil profiles and deposited structures (DepoSim). Gap $G$ is not to scale.

\section{Conclusions}

A simulation study on pattern transfer in nanostencil lithography was carried out to investigate effects of stencil deformation and aperture clogging on deposited surface structures. It was shown that stencil deformation, aperture clogging and consequently pattern distortion can be accurately predicted using conventional modelling tools such as Coventor and Athena. We have been able to relate stencil initial geometry and deposition parameters with geometry of patterned structures which allows assessment of pattern resolution and process window definition.

The accuracy of the models depends on the quality of available material data. It was confirmed that negative effects of stress-induced deformation of stencils can be dealt with via optimal design of corrugation structures which significantly reduces the mask deformation (up to $96 \%$ ) and subsequently translates into improved deposited stencil patterns. Such stencil stabilization enables multiple usage of the stencils and much better control over the pattern definition. Box-type corrugation was found optimal among the geometries considered. Comparison of deflection values of unstabilized and corrugated cantilever membranes showed a good agreement between simulated and measured results. It was also found that the largest reduction in stencil deformation providing superior pattern definition is achieved with corrugation rims located perpendicular to the direction of increasing deformation or "deformation isocontours". In reality, the deformation profile is highly dependent on geometry of structures and the profile alters with the introduction of each series of corrugation rings. Therefore, the design of stabilization structures for real stencils is an iterative process. The design involves iterations of modelling the geometry of stabilization structures followed by re-simulating the deformation profile of the modified structure until the target reduction of deformation or pattern resolution is reached. These "guidelines" can be used as practical design rules for fabrication of stable large area ("full scale") purpose-designed stencil membranes.
Study on the processes leading to the occurrence of clogging shows the influence of surface diffusion to be the most important factor. Direct material deposition on the aperture sidewalls does not lead to a significant reduction in the aperture width.

Combined modelling of clogging phenomenon with gradually evolving stencil deformation, also presented in the paper, was shown to accurately predict the shape of surface structures and can be applied for the estimation of the maximum allowed thickness of deposited layer (feature height) which ultimately defines stencil lifetime.

\section{Acknowledgement}

This Project is funded by EC-funded Project NaPa (contract no. NMP4-CT-2003-500120).

\section{References}

[1] M.A.F. van den Boogaart et al., J. Vac. Sci. Technol. B 22 (6) (2004) 3174-3177.

[2] M. Graff, S.K. Moharty, E. Moss, A.B. Frazier, JMEMS 13 (6) (2004) 956-962.

[3] S. Egger, A. Ilie, Y. Fu, J. Chongsathien, D.-J. Kang, M.E. Welland, Nano Lett. 5 (1) (2005) 15-20.

[4] P.L. Reu, R.L. Engelstad, E.G. Lovell, Microelectron Eng. 69 (2003) 420-428.

[5] R. Tejeda et al., Microelectron Eng. 46 (1999) 481-484.

[6] J. Mackerle, Modelling Simul. Mater. Sci. Eng. 13 (2005) 123-158.

[7] E. Cotte, E. Lovell, R. Engelstad, Microelectron Eng. 57-58 (2001) 461-466.

[8] G. Frisque, R. Tejeda, R. Engelstad, E. Lovell, Microelectron Eng. 53 (2000) 623-626.

[9] Mandar M. Deshmukh, D.C. Ralph, M. Thomas, J. Silcox, Appl. Phys. Lett. 75 (1999) 1631.

[10] M. Koelbel, R.W. Tjerkstra, J. Brugger, C.J.M. van Rijn, W. Nijdam, J. Huskens, D.N. Reinhoudt, Nano Lett. 2 (12) (2002) 1339.

[11] Z. Racz, J. He, S. Srinivasan, W. Zhao, A. Seabaugh, K. Han, P. Ruchhoeft, J. Wolfe, J. Vac. Sci. Technol. B 22 (2004) 74.

[12] M. Koelbel, R.W. Tjerkstra, G.M. Kim, J. Brugger, C.J.M. van Rijn, W. Nijdam, J. Huskens, D.N. Reinhoudt, Adv. Funct. Mater. 13 (3) (2003) 219.

[13] M. Ohring, The Materials Science of Thin Films, Academic press, London, 1992.

[14] K.-S. Chen et al., Thin Solid Films 434 (2003) 190-202.

[15] A. Tarraf et al., J. Micromech. Microeng. 14 (2004) 317-323.

[16] J.A. Thornton, D.W. Hoffman, Thin Solid Films 171 (1) (1989) 5-31.

[17] W. Fang, J.A. Wickert, J. Micromech. Microeng. (5) (1995) 276-281.

[18] R.L. Engelstad et al., Microelectron Eng. 78-79 (2005) 404-409.

[19] A. Molfese, A. Mehta, A. Witrvrouw, Sens. Actuat. A118 (2005) 313-321.

[20] T.G. Bifano et al., JMEMS 11 (5) (2002) 592-597.

[21] CoventorWare version 2005, Reference Guides and Tutorials.

[22] M. Godin et al., Appl. Phys. Lett. 79 (4) (2001) 551-553.

[23] J.E. Sader et al., Rev. Sci. Instrum. 66 (1995) 3789.

[24] H. Baltes et al., CMOC-MEMS, Wiley-VCH, Weinhem, 2005.

[25] R. Whiting, M.A. Angadi, Meas. Sci. Technol. 1 (1990) 662-664.

[26] M.A.F. van den Boogaart, M. Lishchynska, L.M. Doeswijk, J.C. Greer, J. Brugger, Sens. Actuat. A 130-131 (2006) 568-574.

[27] M. Lishchynska, M.A.F. van den Boogaart, J. Brugger, L. Doeswijk, Simulation Study on Mechanical Stabilization of MEMS Stencils for Nanolithography, Micromechanics Europe, September 4-6, 2005, Gothenburg, Sweden. 
[28] http://www.tyndall.ie/research/computational-modelling-group/ vbourenkov.html.

[29] A. Ludwig, J. Cao, J. Brugger, I. Takeuchi, Measur. Sci. Technol. 16 (2005) 111.

[30] ATHENA User's Manual, SILVACO International, Santa Clara, CA 95054, USA (2002).

[31] U. Hansen, S. Rodgers, K.F. Jensen, Phys. Rev. B 62 (1996) 2869.

[32] Robert B. Walker, Joel D. Kress, David E. Hanson, Arthur F. Voter, J. Tinka Gammel, Michael E. Coltrin, Pauline Ho, Modeling the
Growth of Thin Films in Complex 3D Geometrical Structures. T-12 Project Reports, Los Alamos Nat. Lab., USA, 2000.

[33] J.F. Groves. Directed Vapor Deposition. Ph.D. dissertation, University of Virginia, USA (1998).

[34] L.I. Maissel, R. Glang, Handbook of Thin Film Technology, McGraw-Hill Book Company, New York, 1970.

[35] T. Asano, N. Uetake, K. Suzuki, J. Nucl. Sci. Tech. 29 (12) (1992) 1194.

[36] R. Stumpf, M. Scheffler, Phys. Rev. B 53 (1996) 4958. 